\title{
Age-related anatomical changes of spinal nerves and its application to the neuropathic pain model in rat
}

\section{Hiroki Tateiwa, Takashi Kawano, Masataka Yokoyama \\ Department of Anesthesiology and Intensive Care Medicine, Kochi Medical School}

\section{Background}

- Transection of the spinal nerves of L5 and L6 in a spinal nerve ligation (SNL) model is a widely used animal model of neuropathic pain.

- While in most previous studies, SNL has been conducted in young animals, it remains unknown whether SNL could be applied in aged animals.

- The aim of this study is to investigate how aging alters the SNL procedures and to develop a novel technique for SNL model in aged rats.

\section{Materials and Methods}

$>$ Aged male Sprague-Dawley rats (19-24 months old, weighting 650-730g) were used.

- Experiment 1) Analysis of age-related neuroanatomical changes;

After the rats were sacrificed by decapitation under isoflurane anesthesia $(n=5)$, the skin and muscle were removed to expose the nerves of the lumbosacral plexus up to the inter-vertebral foramina. The sciatic nerve was identified at its

departure from the pelvis. The related the vertebral, sacral, and medial iliac bone were also dissected.

\section{Experiment 2) Sensory testing:}

A total of 20 aged male rats were randomly divided into two groups.

Group 1 ( $n=10)$; SNL model with the usual technique in which the L6 transverse process was removed to visually identify the L4 and L5 spinal nerves.

Group 2 ( $\mathrm{n}=10)$; SNL model using a novel technique that identified the L5 spinal nerve under the fascia between the L5 and L6

transverse process without removing it.
Eight and 15 days after SNL surgery, sensory testing for dete

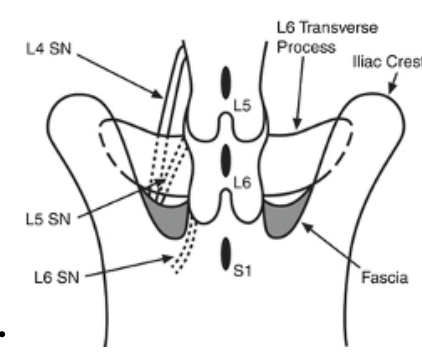

After sensory testings, autopsy was performed on all rats in order to confirm the accuracy of SNL procedures.

\section{$\underline{\text { Result and Discussion }}$}

Experiment 1)
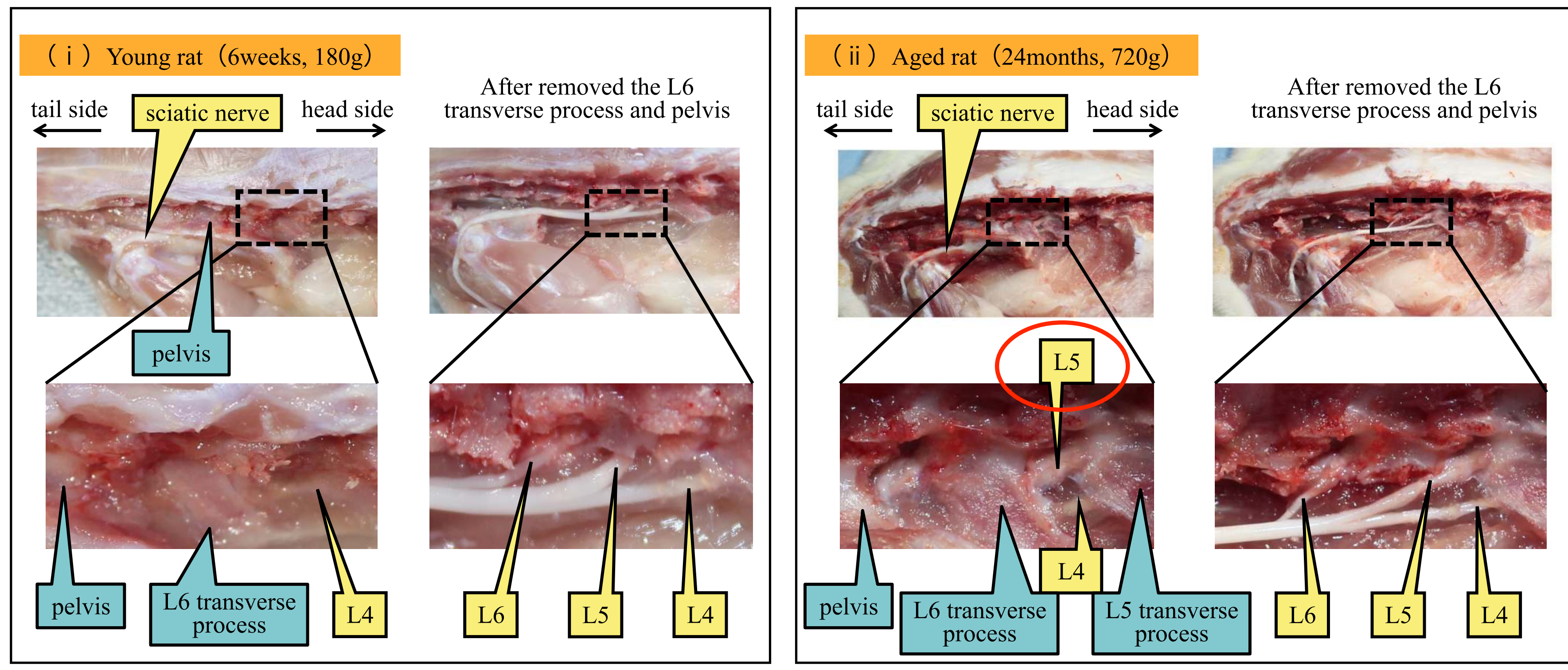

The L4 and L5 spinal nerves were identified under the fascia between the L5 and L6 transverse process in all aged rats.

Therefore, the L5 spinal nerve is identified without removing the L6 transverse process, which is particularly a difficult procedure for aged rats.

\section{Experiment 2)}

- The L4 spinal nerve were injured when the L6 transverse process was removed and developing paralysis in 2 of the 10 rats of Group 1.

- In contrast, in Group 2, L5 spinal nerve was successfully identified and L4 nerve was not injured in all rats.

- No significant difference in hyperalgesia-like behavior was found between the two groups $(\mathrm{p}=0.6176$, Fisher's exact test $)$.

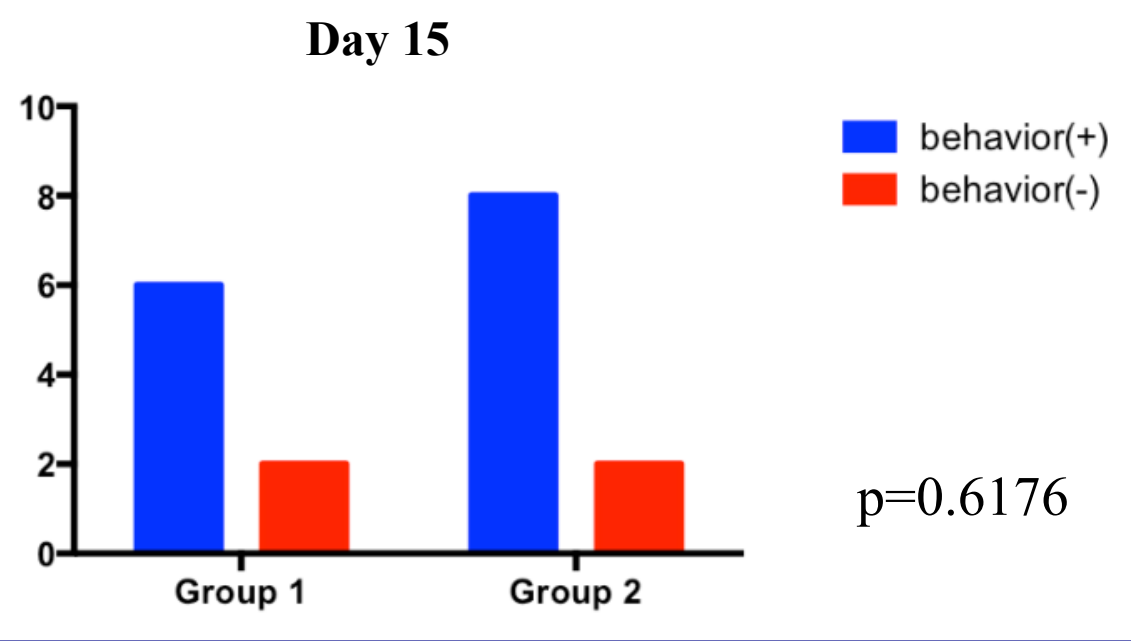

\section{Conclusion}

Our novel technique for SNL model in aged rats may be a promissory approach in studies regarding the age-related pathogenic changes of the neuropathic pain. 